\title{
Practice of Epidemiology
}

\section{An Investigation of Selection Bias in Estimating Racial Disparity in Stroke Risk Factors}

\author{
The REGARDS Study
}

\section{Leann Long*, George Howard, Dustin M. Long, Suzanne Judd, Jennifer J. Manly, Leslie A. McClure, Virginia G. Wadley, Monika M. Safford, Ronit Katz, and M. Maria Glymour}

* Correspondence to Dr. D. Leann Long, Department of Biostatistics, School of Public Health, University of Alabama at Birmingham, 1720 2nd Avenue S, Birmingham, AL 35294 (e-mail: leannl@uab.edu).

Initially submitted June 6, 2018; accepted for publication November 9, 2018.

\begin{abstract}
Selection due to survival or attrition might bias estimates of racial disparities in health, but few studies quantify the likely magnitude of such bias. In a large national cohort with moderate loss to follow-up, we contrasted racial differences in 2 stroke risk factors, incident hypertension and incident left ventricular hypertrophy, estimated by complete-case analyses, inverse probability of attrition weighting, and the survivor average causal effect. We used data on 12,497 black and 17,660 white participants enrolled in the United States (2003-2007) and collected incident risk factor data approximately 10 years after baseline. At follow-up, $21.0 \%$ of white participants and $23.0 \%$ of black participants had died; additionally $22.0 \%$ of white participants and $28.4 \%$ of black participants had withdrawn. Individual probabilities of completing the follow-up visit were estimated using baseline demographic and health characteristics. Adjusted risk ratio estimates of racial disparities from complete-case analyses in both incident hypertension $(1.11,95 \%$ confidence interval: $1.02,1.21)$ and incident left ventricular hypertrophy $(1.02,95 \%$ confidence interval: $0.84,1.24)$ were virtually identical to estimates from inverse probability of attrition weighting and survivor average causal effect. Despite racial differences in mortality and attrition, we found little evidence of selection bias in the estimation of racial differences for these incident risk factors.
\end{abstract}

hypertension; inverse probability weights; left ventricular hypertrophy; racial disparity; selection bias; survivor average causal effect

Abbreviations: CI, confidence interval; IPAW, inverse probability of attrition weighting; LVH, left ventricular hypertrophy; REGARDS, Reasons for Geographic and Racial Differences in Stroke; SACE, survival average causal effect.

In epidemiologic studies assessing incident disease development, traditional statistical analyses are performed only for those participants with disease measurement at follow-up visits (i.e., completers), necessarily excluding those participants lost to attrition (1). However, this approach can introduce bias if the causal effect in participants excluded is inherently different from the effect among participants included in the analyses (i.e., in the context of treatment effect heterogeneity) or if the selection process is influenced by the exposure of interest and another predictor of disease (i.e., in the context of collider bias) (1-4). This selection bias can be a particular issue in longitudinal cohort studies, where the likelihood of attrition increases over time (5). Although death is fundamentally different from standard missing outcome scenarios (6), study withdrawal and death over the follow-up period both potentially introduce bias in estimation of exposure-outcome relationships.

While recent epidemiologic work has advanced knowledge of disease etiology, racial disparities in disease risk persist, and these disparities are marked with respect to stroke and hypertension (7-9). Racial differences in mortality (10-12) imply potential selection bias by race in longitudinal studies. Specifically, estimation of racial disparities in incident disease might be subject to bias if the missingness mechanism disproportionately affects one racial group through selective survival and attrition (13). Simulation studies have shown that this bias can be large in apparently plausible scenarios (13) but only if selection probability is determined by a nonmultiplicative interaction between race and other risk factors for the outcome. To date, there is 
limited empirical evidence from real cohorts on whether such scenarios are likely to occur.

One approach used to address potential selection bias due to study attrition is inverse probability of attrition weighting (IPAW), where participants with follow-up data (which we refer to as "complete case") are weighted to reflect the original baseline population or another population of interest $(2,14)$. This approach estimates the probability of attrition before followup measurement using all cohort participants. Inverses of these attrition probabilities are then applied in analyses of completely observed participants, a process that up-weights complete participant profiles that are underrepresented in observed follow-up data and down-weights those that are overrepresented in the observed data $(2,14)$. This approach can incorporate multiple reasons for attrition, such as death and withdrawal during followup. The ability to consider these different attrition processes provides great flexibility, given that baseline factors associated with death and associated with withdrawal might differ substantially (15). Once probabilities of attrition for each reason have been estimated, they can be multiplied to provide an overall estimated probability of observation, (that is, probability of no attrition) (15). The IPAW estimate can be interpreted as the effect estimate that would have been observed had the cohort not experienced attrition $(2,14,15)$. An alternative interpretation is that weighting recreates the distribution of covariates to mirror the original population and thereby corrects spurious associations that might have been introduced by the selection process (15).

IPAW reflecting no attrition compared with the baseline population has been criticized because the outcome value for people who did not survive to follow-up assessment is undefined, so it is argued that estimating treatment effect in people who died is conceptually incoherent $(6,16-18)$. The survivor average causal effect (SACE) provides an appealing alternative. Distinct from the IPAW analytical approach, SACE is interpreted as the exposure effect among participants who would be complete cases regardless of factors for which the selection bias occurs (6). One calculation of SACE employs the estimated probability of attrition as an additional covariate in the analytical model of interest, conveniently providing a test for the null hypothesis of no selection bias (6).

We are interested in estimation of racial disparities for incident stroke risk factors, and how selection bias might affect these estimates. Our conceptual diagram (Figure 1) reflects how attrition could bias racial disparity estimates; here $L$ are covariates not plausibly affected by race, and $C$ are covariates that might influence selection and are potential consequences of race. Therefore, we used both IPAW and SACE methodologies to account for potential selection bias in a large cohort study and compared these with results from uncorrected analyses based only on respondents who completed the follow-up visit. Our goal was to assess whether either of these sophisticated methods to address selection bias substantially alters apparent racial differences in stroke risk factors. We selected 2 stroke risk factors with differing likely impact: hypertension and left ventricular hypertrophy (LVH). Hypertension was selected because it is a common risk factor anticipated to have a small impact on the risk of death during follow-up (individuals can live years with hypertension). $\mathrm{LVH}$ was selected because it is a less common risk factor, but the potential impact of $\mathrm{LVH}$ on the risk of death during follow-up could be large $(19,20)$.

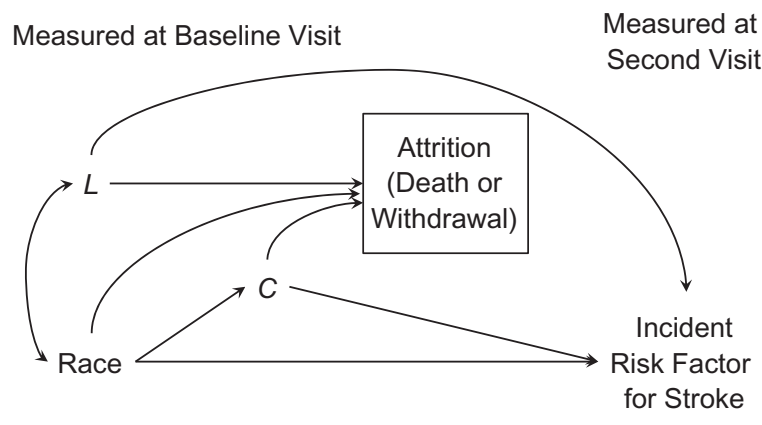

Figure 1. Directed acyclic graph depicting a hypothesized data structure, used in an analysis of data from Reasons for Geographic and Racial Differences in Stroke, United States, 2003-2016. This graph represents the assumptions that a set of covariates $L$ (age, sex, and region) are determined concurrently with race and might be associated due to an unspecified common prior cause (represented by the double-headed arrow), and a set of covariates $C$ (education, income, self-reported health, smoking status, relationship status, health insurance, alcohol use, body mass index category, exercise frequency, rurality, neighborhood socioeconomic score, aspirin use, atrial fibrillation, history of coronary artery disease, history of myocardial infarction, history of transient ischemic attack, diabetes status at baseline, systolic blood pressure, diastolic blood pressure, and $\mathrm{R}$ wave $\mathrm{a} \mathrm{V}_{\mathrm{L}}$ ), which are potentially influenced by race, influence both attrition and incident stroke risk factors (our outcome of interest). Additionally, race might have a direct effect on attrition. The substantive question of interest in the analysis is the effect of race on incident stroke risk factors. The box around attrition indicates that all analyses are by necessity conditioned on this variable. In the sample of individuals who completed the second visit, race might be spuriously associated with $L$ or $C$ due to selective survival or withdrawal. Because race influences incident stroke risk factors in part via covariates $C$, analyses conditioned on $C$ would not estimate the total effect of race on incident stroke risk factors.

\section{METHODS}

\section{Study population}

The Reasons for Geographic and Racial Differences in Stroke (REGARDS) cohort is a national, population-based study of 30,239 community-dwelling participants from the contiguous United States who self-identified as non-Hispanic black or white, with oversampling from the southeastern Stroke Belt and among black persons; the study design and objectives are described in more detail elsewhere (21). Participants were recruited between 2003 and 2007, with inclusion of those age 45 years or older, using commercially available contact information. At enrollment, participants completed detailed surveys and clinical measures, collected via phone and in-home visits performed by technicians trained to the study protocol (21). The study team continues to contact each participant or designated proxy every 6 months by phone for information about stroke events, stroke symptoms, hospitalizations, and death. The study team regularly uses the National Death Index to assess whether participants have died without being detected through regular contact. These brief regular communications allow for time-to-event analysis of adjudicated outcomes such as death, stroke, and coronary heart disease events (22-24), but assessment of many stroke risk factors requires physical examination. Therefore, a second in-person examination of the 
cohort was conducted approximately 10 years after baseline assessment (2013-2016), with goals including assessment of racial and geographic disparities in the development of stroke risk factors. At the time of this second visit, $21.8 \%$ of the original REGARDS participants had died, and an additional $24.7 \%$ declined participation in the second in-home visit (referred to as "withdrawal" henceforth). Retention rates are similar to those of other cohort studies (25-27). In REGARDS, 16,146 (53.5\%) participants completed the follow-up visit, providing researchers with the opportunity to examine a multitude of health status changes, including incident stroke risk factors. Institutional review boards at all participating institutions approved the study, and all participants provided written informed consent.

\section{Outcome assessment}

As important stroke risk factors, 2 cardiovascular outcomes of interest are incident hypertension and incident LVH. Hypertension was assessed at both baseline and follow-up visits and is defined as either self-reported use of medication to control blood pressure or measured systolic blood pressure of $\geq 140 \mathrm{~mm} \mathrm{Hg}$ or diastolic blood pressure of $\geq 90 \mathrm{~mm} \mathrm{Hg}$; blood pressure was the mean of 2 manual measures taken after the participant had been seated for 5 minutes. LVH was defined using the Sokolow-Lyon criteria from electrocardiogram performed at both visits (28). Incident hypertension and $\mathrm{LVH}$ were defined as presence of the risk factor at follow-up among participants without the risk factor at baseline.

\section{Statistical analysis}

Through IPAW, weights are used to create a pseudopopulation, a hypothetical cohort had the attrition not occurred. We performed IPAW analysis relative to 2 pseudopopulations of interest: 1) all REGARDS participants who were not known to be deceased by the time of the expected 10-year follow-up; and 2) all analyzed REGARDS participants. These 2 populations, respectively, represent the REGARDS cohort, assuming either 1) no study withdrawal or 2) both no study withdrawal and no death. Both pseudopopulations were used because we are investigating the potential impact that selection bias from either death or withdrawal might have in estimating racial disparities in incident risk factors in REGARDS, particularly if race is strongly associated with either attrition mechanism.

All participants were coded to indicate a completed followup visit (complete case), study withdrawal, or death. The probabilities of no attrition were predicted through logistic regression models using baseline participant characteristics. Specifically,

$$
\begin{aligned}
& P\left(S_{i}=0\right)=P\left(D_{i}=0, W_{i}=0\right) \\
& \quad=P\left(D_{i}=0\right) P\left(W_{i}=0 \mid D_{i}=0\right)
\end{aligned}
$$

where $S_{i}, W_{i}$, and $D_{i}$ are indicators of attrition, withdrawal, and death for the $i$ th participant.

Because we are not willing to assume that survival and withdrawal are influenced by the same factors, we modeled $P\left(D_{i}=0\right)$ and $P\left(W_{i}=0 \mid D_{i}=0\right)$ separately. Specifically,

$$
\begin{gathered}
\operatorname{Logit}\left(P\left(D_{i}=0 \mid \text { Race }_{i}, L_{i}, C_{i}\right)\right. \\
=\alpha_{\mathrm{D}}+\beta_{\mathrm{D}} I\left(\text { Race }_{i}=\text { black }\right) \\
+\theta_{\mathrm{D}} L_{i}+\gamma_{\mathrm{D}} C_{i}+\delta_{\mathrm{D}} C_{i}{ }^{*} \\
\\
\operatorname{Logit}\left(P\left(W_{i}=0 \mid \text { Race }_{i}, L_{i}, C_{i}, D_{i}=0\right)\right. \\
=\alpha_{\mathrm{W}}+\beta_{\mathrm{W}} I\left(\text { Race }_{i}=\text { black }\right) \\
+\theta_{\mathrm{W}} L_{i}+\gamma_{\mathrm{W}} C_{i}+\delta_{\mathrm{W}} C_{i}{ }^{*},
\end{gathered}
$$

where $L_{i}$ are predictors of attrition not plausibly affected by race, $C_{i}$ predictors of attrition that occurred later in life and might be influenced by race (Figure 1), and $C_{i}^{*}$ includes all pairwise interactions between race, $L_{i}$, and $C_{i}$. The quantities $\alpha, \beta, \theta, \gamma$, and $\delta$ and accompanying subscripts $\mathrm{W}$ and $\mathrm{D}$ in equation 2 reflect the distinct model parameters for withdrawal and death models.

See Table 1 for the list of covariates considered and the coding of each as modeled. Assumed to occur concurrent with assessment of race and potentially confound effect estimation, we define $L$ to include age, sex, and region. The additional variables are included in $C$, which are assumed to be potentially influenced by race and therefore not confounders of race. Sensitivity analyses additionally included cognitive function (Six-Item Screener score of $\geq 4$ ) on the subset of participants with measurement at baseline data collection (29).

Because we examined age continuously, up to a cubic term of age was included in each predictive model for increased weight model fit. Each weight model was examined using Hosmer-Lemeshow goodness-of-fit $\chi^{2}$ test (30) and Hinkley's method of assessing link function appropriateness $(1,31)$. Given that the attrition mechanism should be a function of variables related to both exposure and outcome for selection bias to be present, we selected predictors measured at baseline that are thought to be related to race or incident risk factor and maximize model predictive ability, measured through the $C$ statistic (1). For IPAW prediction models, we used backward model selection using all pairwise interactions in $C_{i}^{*}$, including those between race and each of the predictors in $C_{i}$ to account for potential collider bias $(4,13)$. To examine the impact of weight modeling decisions on the racial disparity estimates, a variety of weight models were examined, specifically assessing the importance of decisions of whether to exclude participants with prevalent disease before or after weight estimation and selection methods for interaction terms.

The predicted probabilities (equation 2) were then multiplied to obtain the estimated $P\left(S_{i}=0 \mid \mathrm{Race}_{i}, L_{i}, C_{i}\right)$ (equation 1), which were then inverted and stabilized to create weights used in standard analytical methods $(4,14)$. Stabilized weights were created by multiplying the nonstabilized weights by $P\left(S_{i}=0 \mid\right.$ Race $\left._{i}, L_{i}\right)$. The premise behind the stabilization is to reduce potential undue influence of the often highly variable nonstabilized weights (14). While both stabilized and nonstabilized weights can provide unbiased estimates, reduction of extremely large weights can provide gains in efficiency $(14,15)$. After weight estimation, we used modified Poisson regression models, applying stabilized weights, to estimate the risk ratio between race and each incident risk factor (32).

To estimate the SACE, we used the 2-stage estimation technique outlined in Tchetgen Tchetgen et al. (6). First, separate 
Table 1. Baseline Characteristics of Participants, Used Separately to Predict Attrition, Unadjusted Odds Ratios, and $C$ statistics for Death and Withdrawal ( $n=29,947)$, Reasons for Geographic and Racial Differences in Stroke, United States, 2003-2016

\begin{tabular}{|c|c|c|c|c|c|c|c|c|c|c|}
\hline \multirow[b]{2}{*}{ Baseline Predictor } & \multirow{2}{*}{$\begin{array}{l}\text { Baseline } \\
\text { No. }\end{array}$} & \multirow[b]{2}{*}{$\%$} & \multicolumn{4}{|c|}{ Withdrawal (Among Alive) } & \multicolumn{4}{|c|}{ Death } \\
\hline & & & $\begin{array}{c}\text { \% Lost From } \\
\text { Baseline }\end{array}$ & $\begin{array}{l}\text { Unadjusted } \\
\text { OR }\end{array}$ & $95 \% \mathrm{Cl}$ & $\begin{array}{l}\text { Unadjusted } C \\
\text { Statistic }\end{array}$ & $\begin{array}{l}\text { \% Died From } \\
\text { Baseline }\end{array}$ & $\begin{array}{l}\text { Unadjusted } \\
\text { OR }\end{array}$ & $95 \% \mathrm{Cl}$ & $\begin{array}{l}\text { Unadjusted } \\
\text { C Statistic }\end{array}$ \\
\hline Race & & & & & & 0.550 & & & & 0.514 \\
\hline Black & 12,418 & 41 & 36.8 & 1.51 & $1.43,1.59$ & & 23.0 & 1.12 & $1.06,1.19$ & \\
\hline White & 17,529 & 59 & 27.9 & 1.00 & Referent & & 21.0 & 1.00 & Referent & \\
\hline $\mathrm{Age}^{\mathrm{a}}$ & & & & 0.93 & $0.90,0.96$ & 0.521 & & 2.84 & $2.74,2.94$ & 0.745 \\
\hline Systolic blood pressure ${ }^{b}$ & & & & 1.01 & $1.00,1.01$ & 0.506 & & 1.10 & $1.10,1.11$ & 0.595 \\
\hline Diastolic blood pressure ${ }^{\mathrm{b}}$ & & & & 0.95 & $0.93,0.96$ & 0.529 & & 0.96 & $0.95,0.97$ & 0.522 \\
\hline R wave $\mathrm{aV}_{\mathrm{L}}{ }^{\mathrm{c}}$ & & & & 1.00 & $0.99,1.01$ & 0.502 & & 1.05 & $1.04,1.06$ & 0.542 \\
\hline Sex & & & & & & 0.518 & & & & 0.563 \\
\hline Male & 13,445 & 45 & 2.97 & 0.86 & $0.82,0.91$ & & 26.6 & 1.65 & $1.57,1.75$ & \\
\hline Female & 16,502 & 55 & 32.9 & 1.00 & Referent & & 17.9 & 1.00 & Referent & \\
\hline Region $^{d}$ & & & & & & 0.514 & & & & 0.516 \\
\hline Stroke Belt & 10,364 & 35 & 33.1 & 1.13 & $1.06,1.20$ & & 22.1 & 0.97 & $0.91,1.03$ & \\
\hline Stroke Buckle & 6,276 & 21 & 30.9 & 1.02 & $0.95,1.10$ & & 19.7 & 0.84 & $0.78,0.90$ & \\
\hline Outside the Stroke Belt & 13,307 & 44 & 30.5 & 1.00 & Referent & & 22.6 & 1.00 & Referent & \\
\hline General health $^{\mathrm{e}}$ & & & & & & 0.550 & & & & 0.621 \\
\hline Excellent & 4,778 & 16 & 27.0 & 1.00 & Referent & & 12.8 & 1.00 & Referent & \\
\hline Very good & 9,097 & 30 & 28.5 & 1.08 & $0.99,1.17$ & & 16.5 & 1.35 & $1.22,1.50$ & \\
\hline Good & 10,488 & 35 & 33.5 & 1.36 & $1.26,1.48$ & & 23.1 & 2.05 & $1.87,2.26$ & \\
\hline Fair & 4,467 & 15 & 38.0 & 1.66 & $1.50,1.84$ & & 33.1 & 3.39 & $3.05,3.76$ & \\
\hline Poor & 1,056 & 4 & 43.0 & 2.04 & $1.70,2.44$ & & 46.9 & 6.03 & $5.20,6.99$ & \\
\hline Educational level $^{\mathrm{e}}$ & & & & & & 0.584 & & & & 0.592 \\
\hline Less than high school & 3,764 & 13 & 44.9 & 2.58 & $2.35,2.84$ & & 37.2 & 3.11 & $2.86,3.39$ & \\
\hline High school & 7,750 & 26 & 36.1 & 1.79 & $1.67,1.93$ & & 22.8 & 1.56 & $1.45,1.68$ & \\
\hline Some college & 8,036 & 27 & 32.6 & 1.54 & $1.43,1.65$ & & 21.2 & 1.41 & $1.31,1.52$ & \\
\hline $\begin{array}{l}\text { College graduate or } \\
\text { more }\end{array}$ & 10,397 & 35 & 24.0 & 1.00 & Referent & & 16.0 & 1.00 & Referent & \\
\hline Income level, $\$$ & & & & & & 0.575 & & & & 0.626 \\
\hline$<20,000$ & 5,438 & 18 & 41.9 & 2.26 & $2.05,2.49$ & & 33.0 & 4.57 & $4.09,5.11$ & \\
\hline $20,000-34,999$ & 7,251 & 24 & 33.4 & 1.57 & $1.43,1.72$ & & 26.4 & 3.33 & $2.98,3.71$ & \\
\hline $35,000-74,999$ & 8,848 & 30 & 27.6 & 1.20 & $1.10,1.31$ & & 16.6 & 1.85 & $1.65,2.07$ & \\
\hline$\geq 75,000$ & 4,723 & 16 & 24.2 & 1.00 & Referent & & 9.7 & 1.00 & Referent & \\
\hline Refused & 3,687 & 12 & 35.8 & 1.75 & $1.58,1.94$ & & 24.3 & 2.99 & $2.64,3.37$ & \\
\hline
\end{tabular}




\begin{tabular}{|c|c|c|c|c|c|c|c|c|c|c|}
\hline \multirow[b]{2}{*}{ Baseline Predictor } & \multirow{2}{*}{$\begin{array}{l}\text { Baseline } \\
\text { No. }\end{array}$} & \multirow[b]{2}{*}{$\%$} & \multicolumn{4}{|c|}{ Withdrawal (Among Alive) } & \multicolumn{4}{|c|}{ Death } \\
\hline & & & $\begin{array}{l}\text { \% Lost From } \\
\text { Baseline }\end{array}$ & $\begin{array}{l}\text { Unadjusted } \\
\text { OR }\end{array}$ & $95 \% \mathrm{Cl}$ & $\begin{array}{c}\text { Unadjusted C } \\
\text { Statistic }\end{array}$ & $\begin{array}{l}\text { \% Died From } \\
\text { Baseline }\end{array}$ & $\begin{array}{l}\text { Unadjusted } \\
\text { OR }\end{array}$ & $95 \% \mathrm{Cl}$ & $\begin{array}{l}\text { Unadjusted } \\
\text { C Statistic }\end{array}$ \\
\hline Smoking status $^{\mathrm{e}}$ & & & & & & 0.527 & & & & 0.569 \\
\hline Never & 13,495 & 45 & 30.1 & 1.00 & Referent & & 17.2 & 1.00 & Referent & \\
\hline Past & 11,975 & 40 & 30.1 & 1.00 & $0.94,1.06$ & & 24.4 & 1.55 & $1.46,1.65$ & \\
\hline Current & 4,362 & 15 & 40.4 & 1.57 & $1.45,1.71$ & & 29.0 & 1.96 & $1.81,2.12$ & \\
\hline Relationship status ${ }^{\mathrm{e}}$ & & & & & & 0.540 & & & & 0.570 \\
\hline Single & 1,595 & 5 & 35.7 & 1.37 & $1.22,1.55$ & & 20.5 & 1.12 & $0.98,1.27$ & \\
\hline Married & 17,590 & 59 & 28.8 & 1.00 & Referent & & 18.8 & 1.00 & Referent & \\
\hline Divorced & 4,382 & 15 & 35.2 & 1.34 & $1.24,1.45$ & & 19.2 & 1.03 & $0.94,1.12$ & \\
\hline Widowed & 5,661 & 19 & 35.3 & 1.35 & $1.25,1.45$ & & 33.9 & 2.22 & $2.07,2.37$ & \\
\hline Other & 719 & 2 & 41.9 & 1.78 & $1.51,2.11$ & & 19.8 & 1.06 & $0.88,1.28$ & \\
\hline Health insurance ${ }^{e}$ & & & & & & 0.518 & & & & 0.512 \\
\hline Yes & 27,934 & 93 & 30.7 & 1.00 & Referent & & 22.2 & 1.00 & Referent & \\
\hline No & 1,986 & 7 & 42.5 & 1.67 & $1.51,1.85$ & & 15.5 & 0.64 & $0.57,0.73$ & \\
\hline Alcohol use $^{e}$ & & & & & & 0.530 & & & & 0.542 \\
\hline Yes & 10.966 & 37 & 28.1 & 0.77 & $0.73,0.82$ & & 17.9 & 0.69 & $0.65,0.73$ & \\
\hline No & 18,981 & 63 & 33.5 & 1.00 & Referent & & 23.9 & 1.00 & Referent & \\
\hline $\begin{array}{l}\text { Body mass index } \\
\text { category }^{\dagger}\end{array}$ & & & & & & 0.515 & & & & 0.536 \\
\hline Normal $(<25.0)$ & 7,375 & 25 & 30.5 & 1.00 & Referent & & 25.90 & 1.00 & Referent & \\
\hline $\begin{array}{l}\text { Overweight } \\
\quad(25.0-29.9)\end{array}$ & 10,991 & 37 & 30.5 & 1.00 & $0.93,1.07$ & & 21.0 & 0.76 & $0.71,0.82$ & \\
\hline Obese $(\geq 30.0)$ & 11,428 & 38 & 33.1 & 1.13 & $1.05,1.21$ & & 19.6 & 0.70 & $0.65,0.75$ & \\
\hline Exercise category ${ }^{\mathrm{e}}$ & & & & & & 0.522 & & & & 0.581 \\
\hline None & 10,156 & 34 & 33.6 & 1.00 & Referent & & 29.1 & 1.00 & Referent & \\
\hline 1-3 times per week & 10,613 & 36 & 29.4 & 0.82 & $0.77,0.88$ & & 17.0 & 0.50 & $0.47,0.53$ & \\
\hline$\geq 4$ times per week & 8,735 & 30 & 31.7 & 0.92 & $0.86,0.98$ & & 18.8 & 0.57 & $0.53,0.61$ & \\
\hline Urban group $^{g}$ & & & & & & 0.506 & & & & 0.524 \\
\hline Rural & 2,900 & 11 & 29.1 & 0.89 & $0.81,0.97$ & & 18.2 & 0.75 & $0.68,0.83$ & \\
\hline Mixed & 2,933 & 11 & 31.8 & 1.00 & $0.92,1.10$ & & 18.1 & 0.74 & $0.67,0.82$ & \\
\hline Urban & 21,230 & 78 & 31.7 & 1.00 & Referent & & 23.0 & 1.00 & Referent & \\
\hline $\begin{array}{l}\text { nSES (census-block } \\
\text { level), quartile }\end{array}$ & & & & & & 0.560 & & & & 0.564 \\
\hline 1 & 6,695 & 25 & 37.8 & 1.79 & $1.65,1.95$ & & 27.0 & 1.82 & $1.67,1.98$ & \\
\hline 2 & 6,723 & 25 & 33.7 & 1.50 & $1.38,1.63$ & & 24.1 & 1.56 & $1.44,1.70$ & \\
\hline
\end{tabular}


Table 1. Continued

\begin{tabular}{|c|c|c|c|c|c|c|c|c|c|c|}
\hline \multirow[b]{2}{*}{ Baseline Predictor } & \multirow{2}{*}{$\begin{array}{l}\text { Baseline } \\
\text { No. }\end{array}$} & \multirow[b]{2}{*}{$\%$} & \multicolumn{4}{|c|}{ Withdrawal (Among Alive) } & \multicolumn{4}{|c|}{ Death } \\
\hline & & & $\begin{array}{l}\text { \% Lost From } \\
\text { Baseline }\end{array}$ & $\begin{array}{l}\text { Unadjusted } \\
\text { OR }\end{array}$ & $95 \% \mathrm{Cl}$ & $\begin{array}{l}\text { Unadjusted } C \\
\text { Statistic }\end{array}$ & $\begin{array}{l}\text { \% Died From } \\
\text { Baseline }\end{array}$ & $\begin{array}{c}\text { Unadjusted } \\
\text { OR }\end{array}$ & $95 \% \mathrm{Cl}$ & $\begin{array}{l}\text { Unadjusted } \\
\text { C Statistic }\end{array}$ \\
\hline 3 & 6,715 & 25 & 29.8 & 1.25 & $1.15,1.36$ & & 19.7 & 1.21 & $1.11,1.32$ & \\
\hline 4 & 6,710 & 25 & 25.3 & 1.00 & Referent & & 16.9 & 1.00 & Referent & \\
\hline Coronary artery disease $^{\mathrm{h}}$ & & & & & & 0.505 & & & & 0.598 \\
\hline Yes & 5,297 & 18 & 33.0 & 1.08 & $1.00,1.17$ & & 40.2 & 3.13 & $2.94,3.34$ & \\
\hline No & 24,157 & 82 & 31.3 & 1.00 & Referent & & 17.7 & 1.00 & Referent & \\
\hline Atrial fibrillation $^{\mathrm{h}}$ & & & & & & 0.502 & & & & 0.540 \\
\hline Yes & 2,564 & 9 & 33.0 & 1.08 & $0.97,1.20$ & & 37.1 & 2.34 & $2.15,2.55$ & \\
\hline No & 26,694 & 91 & 31.3 & 1.00 & Referent & & 20.1 & 1.00 & Referent & \\
\hline Diabetes $^{i}$ & & & & & & 0.521 & & & & 0.574 \\
\hline Yes & 6,341 & 22 & 36.1 & 1.30 & $1.22,1.40$ & & 33.1 & 2.20 & $2.06,2.34$ & \\
\hline No & 22,528 & 78 & 30.2 & 1.00 & Referent & & 18.4 & 1.00 & Referent & \\
\hline Hypertension ${ }^{\mathrm{j}}$ & & & & & & 0.522 & & & & 0.578 \\
\hline Yes & 17,720 & 59 & 33.2 & 1.19 & $1.13,1.26$ & & 26.2 & 1.97 & $1.86,2.10$ & \\
\hline No & 12,194 & 41 & 29.4 & 1.00 & Referent & & 15.3 & 1.00 & Referent & \\
\hline Myocardial infarction ${ }^{\mathrm{h}}$ & & & & & & 0.506 & & & & 0.576 \\
\hline Yes & 3,787 & 13 & 34.0 & 1.13 & $1.03,1.24$ & & 41.8 & 3.11 & $2.89,3.34$ & \\
\hline No & 25,659 & 87 & 31.2 & 1.00 & Referent & & 18.8 & 1.00 & Referent & \\
\hline Regular aspirin use $\mathrm{e}^{\mathrm{e}}$ & & & & & & 0.511 & & & & 0.544 \\
\hline Yes & 12,941 & 43 & 30.4 & 0.91 & $0.86,0.97$ & & 25.3 & 1.43 & $1.35,1.51$ & \\
\hline No & 16,989 & 57 & 32.3 & 1.00 & Referent & & 19.2 & 1.00 & Referent & \\
\hline $\begin{array}{l}\text { Transient ischemic } \\
\text { attack }^{\mathrm{e}}\end{array}$ & & & & & & 0.504 & & & & 0.525 \\
\hline Yes & 1,104 & 4 & 36.3 & 1.27 & $1.08,1.49$ & & 40.1 & 2.81 & $2.48,3.18$ & \\
\hline No & 26,770 & 96 & 31.1 & 1.00 & Referent & & 19.3 & 1.00 & Referent & \\
\hline
\end{tabular}

\footnotetext{
$\sum_{c}$ Abbreviations: $\mathrm{Cl}$, confidence interval; $\mathrm{nSES}$, neighborhood socioeconomic status; OR, odds ratio.

a Odds ratios are given for each 10 -year increase.

${ }^{\mathrm{b}}$ Odds ratios are given for each $10-\mathrm{mm} \mathrm{Hg}$ increase.

${ }^{\mathrm{c}}$ Odds ratios are given for each $100-\mathrm{V}$ increase.

${ }^{\mathrm{d}}$ Regions: Stroke Buckle (coastal plain region of North Carolina, South Carolina, and Georgia), Stroke Belt (Alabama, Arkansas, Louisiana, Mississippi, Tennessee, and remainder of North Carolina, South Carolina, and Georgia), and outside the Stroke Belt (remainder of contiguous United States).

e Self-reported.

${ }^{\mathrm{f}}$ Weight $(\mathrm{kg}) /$ height $(\mathrm{m})^{2}$.

${ }^{2}$ Defined by the 2000 US Census.

${ }^{\mathrm{h}}$ Self-reported or electrocardiogram-detected.

'Diabetes was defined as a fasting serum glucose of $\geq 126 \mathrm{mg} / \mathrm{dL}$, a random serum glucose of $\geq 200 \mathrm{mg} / \mathrm{dL}$, or self-reported use of insulin or oral diabetes medications.

j Self-reported use of medications to control blood pressure or measured systolic blood pressure $\geq 140 \mathrm{~mm} \mathrm{Hg}$ or diastolic blood pressure $\geq 90 \mathrm{~mm} \mathrm{Hg}$
} 
predictive models of death and withdrawal were fitted using all participants, but only main effects of predictors in $L$ and $C$ were included so that survival prediction models and analytical models contained the same set of variables (6). As in the IPAW analysis, predicted probabilities of both attrition mechanisms were combined to obtain predicted $P\left(S_{i}=0 \mid\right.$ Race $\left._{i}, L_{i}, C_{i}\right)$ (equation 1). In the second estimation stage, the predicted $P\left(S_{i}=\right.$ 1 | Race $\left._{i}, L_{i}, C_{i}\right) \equiv Q_{i}$ was included as a covariate in the model assessing the relationship between race and incident risk factor. Specifically, we used modified Poisson regression models to estimate risk ratio of racial disparity in the 2 risk factors. The second stage models for incident hypertension and incident $\mathrm{LVH}$ are expressed

$$
\begin{aligned}
\log & E\left(Y_{1 i} \mid \text { Race }_{i}, L_{i}, C_{i}, S_{i}=1\right) \\
= & \alpha_{1}+\beta_{1} I\left(\text { Race }_{i}=\text { black }\right)+\psi_{i} Q_{i} \\
& +\theta_{1} L_{i}+\gamma_{1} C_{i}
\end{aligned}
$$

$$
\begin{aligned}
\log & E\left(Y_{2 i} \mid \text { Race }_{i}, L_{i}, C_{i}, S_{i}=1\right) \\
= & \alpha_{2}+\beta_{2} I\left(\text { Race }_{i}=\text { black }\right)+\psi_{2} Q_{i} \\
& +\theta_{2} L_{i}+\gamma_{2} C_{i},
\end{aligned}
$$

where $Y_{1 i}$ and $Y_{2 i}$ represent incident hypertension and $\mathrm{LVH}$, respectively. The regression coefficients $\psi_{1}$ and $\psi_{2}$ quantify the extent of survival bias (for incident hypertension and incident LVH, respectively) and the test of $H_{0}: \psi_{k}=0, k=(1$ for incident hypertension, 2 for incident $\mathrm{LVH}$ ) can be considered a test for the presence of selection bias (6).

SACE estimation relies on several key assumptions (6). We assume that all common causes of $S$ and $Y_{1}$ and $Y_{2}$ are contained within our confounders $L$ and $C$ and a set of unmeasured confounders $U$. Although permitting the existence of unmeasured confounders $U$ is an appealing feature of this approach to estimating the SACE, the approach is valid only under the assumption that the structure of the relationship between $U_{i}$ and $S_{i}$ follows: $\operatorname{logit}\left(S_{i}=1 \mid \mathrm{Race}_{i}, L_{i}, C_{i}, U_{i}\right)=\alpha U_{i}+f\left(\mathrm{Race}_{i}, L_{i}, C_{i}\right)$. When there is no unmeasured confounding, $\operatorname{logit}\left(S_{i}=1\right.$ l Race $\left._{i}, L_{i}, C_{i}, U_{i}\right)=\operatorname{logit}\left(S_{i}=1 \mid\right.$ Race $\left._{i}, L_{i}, C_{i}\right)$. These assumptions combine to yield that $Y$ is independent of counterfactual survival status, conditional on $L$ and $C$. Additionally, the SACE methodology assumes that race is independent of $U$ given $L$ and $C$ and that impact of survival bias is the same across the distribution of $U$ among $S=0$. Finally we must assume correct model specification (equations $3 a$ and $3 b$ ).

To investigate potential selection bias, we estimated the race association measured by risk ratio for both incident hypertension and LVH using IPAW and SACE methods and compared these results with the complete-case analysis. Confidence intervals used in both IPAW and SACE were obtained through empirical bootstrap $(6,33,34)$. While SACE methods require adjustment for $L$ and $C$ in the final analytical model, use of IPAW circumvents this problem. To facilitate comparison of conventional analyses, IPAW, and SACE estimation, 3 models were considered: Model 1 estimates the unadjusted (crude) association between race and each outcome, model 2 estimates racial disparities in the outcomes adjusting for $L$, and model 3 estimates racial disparities adjusted for both $L$ and $C$. Statistical analyses were performed in SAS, version 9.4 (SAS Institute, Inc., Cary, North Carolina).

\section{RESULTS}

The original REGARDS study sample size was 30,239. After excluding 56 participants with data anomalies (0.19\%) and 236 $(0.78 \%)$ with missing region, education, electrocardiogram $\mathrm{R}$ wave $\mathrm{aV}_{\mathrm{L}}$ measurement, or systolic or diastolic blood pressure, we had 29,947 participants with baseline characteristics to build predictive models for study withdrawal and death (Web Figure 1, available at https://academic.oup.com/aje). The cohort was $41 \%$ black and $45 \%$ male, with $44 \%$ residing outside of the Stroke Belt or Buckle and mean age of 64.8 (standard deviation, 9.4) years. At baseline, $59 \%$ of participants had hypertension and $10 \%$ had LVH. Table 1 displays attrition from withdrawal or death, unadjusted odds ratios of each attrition mechanism, and $C$ statistic for each baseline predictor separately. The most predictive variables for study withdrawal were educational level, income level, and neighborhood socioeconomic status $(35,36)$. Age, income level, and self-reported general health were most individually predictive of death. Although $C$ statistics linking race with withdrawal (0.550; 95\% confidence interval (CI): $0.543,0.557)$ and death $(0.514 ; 95 \%$ CI: $0.507,0.521)$ were statistically different from 0.5 , the magnitude of association was modest.

Combining all baseline variables into collective models for death and withdrawal (Table 2), we observed a $C$ statistic for study withdrawal of $0.636(95 \% \mathrm{CI}: 0.630,0.645)$ and a $C$ statistic for death of 0.830 (95\% CI: $0.825,0.836)$ when adjusting for covariates $L, C$, and those components of $C^{*}$ selected through backward selection (see Web Table 1 for model parameter estimates). Omitting $C^{*}$ for the SACE estimation, $C$ statistics were 0.622 (95\% CI: $0.613,0.630)$ and 0.823 (95\% CI: 0.816, 0.830) for withdrawal and death, respectively.

Examining IPAW weight distributions in Table 2, the range of the nonstabilized (raw) weights was relatively narrow for withdrawal (1.00-4.89) and larger for death (1.00-13.80). Both nonstabilized weight distributions were heavily rightskewed. Those participants with largest weights in the death model were typically over 80 years old at baseline, male, hypertensive, and had urban residence at baseline. Participants with largest withdrawal weights were black, hypertensive, in the lowest income level, in the lowest neighborhood socioeconomic quartile $(35,36)$, and had urban residence at baseline. Note that because these individuals were hypertensive at baseline, they were excluded from analyses where incident hypertension was the outcome.

The follow-up sample consisted of 12,604 participants with complete data. After removing those with missing or prevalent risk factors, analytical sample sizes were 5,530 for incident hypertension analysis and 9,969 for incident $\mathrm{LVH}$ analysis (Web Figure 1). Table 3 provides the risk ratio for black versus white persons, using models $1-3$. From model 2 of incident hypertension adjusted for $L$, black persons had an estimated risk ratio of 1.43 (95\% CI: $1.33,1.54)$ and 1.41 (95\% CI: 1.34 , 1.54) compared with white persons using complete-case analysis and IPAW-stabilized weighting with no death or withdrawal, respectively. In the incident hypertension model 3, which additionally adjusts for $C$, the estimated risk ratio for race 
Table 2. Fit Statistics and Weights According to Attrition Model for Withdrawal, Death, and Combination ( $n=$ 29,947), Reasons for Geographic and Racial Differences in Stroke, United States, 2003-2016

\begin{tabular}{|c|c|c|c|c|c|c|c|}
\hline \multirow[b]{2}{*}{ Attrition Model } & \multicolumn{3}{|c|}{ Fit Statistic } & \multicolumn{2}{|c|}{ Nonstabilized Weight } & \multicolumn{2}{|c|}{ Stabilized Weight $^{a}$} \\
\hline & $\%$ Discordant & C Statistic & $\begin{array}{c}\text { Hosmer-Lemeshow } \\
P \text { Value }\end{array}$ & Mean (SD) & Range & Mean (SD) & Range \\
\hline \multicolumn{8}{|l|}{ IPAW } \\
\hline Death & 17.0 & 0.830 & 0.34 & $1.25(0.51)$ & $1.00-13.80$ & $1.05(0.16)$ & $0.12-2.34$ \\
\hline Withdrawal & 36.4 & 0.636 & 0.79 & $1.46(0.25)$ & $1.00-4.89$ & $1.02(0.13)$ & $0.30-1.70$ \\
\hline Combination & & & & $1.86(1.01)$ & $1.02-27.15$ & $1.07(0.24)$ & $0.11-2.19$ \\
\hline \multicolumn{8}{|l|}{ SACE } \\
\hline Death & 17.7 & 0.823 & 0.42 & & & & \\
\hline Withdrawal & 37.8 & 0.622 & 0.43 & & & & \\
\hline
\end{tabular}

Abbreviations: IPAW, inverse probability of attrition weighting; SACE, survivor average causal effect; SD, standard deviation.

a Stabilized weight: race, age, sex, and region in numerator.

is 1.11 (95\% CI: $1.02,1.21)$ in complete-case analysis, 1.11 (95\% CI: $1.03,1.23)$ using stabilized IPAW with no death or withdrawal, and the estimated SACE is 1.15 (95\% CI: 1.05, 1.26). Incident $\mathrm{LVH}$ risk ratio estimates are similarly comparable across models and analytical methods. For SACE methodology, estimates of $\psi$ were not significantly different from the null for either the hypertension $\left(\psi_{1}=-1.29 ; 95 \% \mathrm{CI}:-2.61\right.$,
$0.02)$ or $\mathrm{LVH}\left(\psi_{2}=1.10\right.$; $\left.95 \% \mathrm{CI}:-1.58,3.88\right)$ models, indicating a lack of evidence for selection bias.

In sensitivity analysis, we evaluated whether inclusion of cognitive function among a subset of participants $(n=24,257)$ improved predictive ability of either death or withdrawal models. The additional information did not improve predictive ability; both IPAW and SACE estimates of racial

Table 3. Estimated Risk Ratios for Black Versus White Participants for Each Cardiovascular Outcome According to Analytical Technique (Hypertension, $n=5,530 ; \mathrm{LVH}, n=9,969$ ), Reasons for Geographic and Racial Differences in Stroke, United States, 2003-2016

\begin{tabular}{|c|c|c|c|c|c|c|c|c|c|c|c|c|}
\hline \multirow{3}{*}{ Analytical Technique } & \multicolumn{6}{|c|}{ Incident Hypertension } & \multicolumn{6}{|c|}{ Incident LVH } \\
\hline & \multicolumn{2}{|c|}{ Model $1^{a}$} & \multicolumn{2}{|c|}{ Model $2^{\mathrm{b}}$} & \multicolumn{2}{|c|}{ Model $3^{c}$} & \multicolumn{2}{|c|}{ Model $1^{\mathrm{a}}$} & \multicolumn{2}{|c|}{ Model $2^{\mathrm{b}}$} & \multicolumn{2}{|c|}{ Model $3^{c}$} \\
\hline & 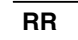 & $95 \% \mathrm{Cl}$ & $\mathbf{R R}$ & $95 \% \mathrm{Cl}$ & $\mathbf{R R}$ & $95 \% \mathrm{Cl}$ & $\begin{array}{l}\mathbf{R} \\
\end{array}$ & $95 \% \mathrm{Cl}$ & $\mathbf{R R}$ & $95 \% \mathrm{Cl}$ & $\begin{array}{l}\mathbf{R R} \\
\mathbf{n}\end{array}$ & $95 \% \mathrm{Cl}$ \\
\hline \multicolumn{13}{|c|}{ ( } \\
\hline \multicolumn{13}{|l|}{ Unstabilized weights } \\
\hline \multicolumn{13}{|l|}{ Stabilized weights ${ }^{\mathrm{e}}$} \\
\hline Withdrawal weights & 1.40 & $1.34,1.52$ & 1.42 & $1.35,1.54$ & 1.12 & $1.03,1.22$ & 1.49 & $1.27,1.74$ & 1.53 & $1.30,1.80$ & 1.03 & $0.82,1.26$ \\
\hline Withdrawal or death weights & 1.40 & $1.32,1.53$ & 1.41 & $1.34,1.54$ & 1.11 & $1.03,1.23$ & 1.49 & $1.16,1.62$ & 1.52 & $1.18,1.65$ & 1.02 & $0.78,1.20$ \\
\hline \multicolumn{13}{|l|}{ SACE $^{d}$} \\
\hline $\begin{array}{l}\text { Accounting for death and } \\
\text { withdrawal }\end{array}$ & & & & & 1.15 & $1.05,1.26$ & & & & & 1.00 & $0.80,1.22$ \\
\hline
\end{tabular}

Abbreviations: Cl, confidence interval; IPAW, inverse probability of attrition weighting; LVH, left ventricular hypertrophy; RR, risk ratio; SACE, survivor average causal effect.

${ }^{\text {a }}$ Model 1 had no adjustments.

${ }^{\mathrm{b}}$ Model 2 adjusted for $L$ (age, sex, and region).

${ }^{\mathrm{c}}$ Model 3 additionally adjusted for $C$ (age ${ }^{2}$, age ${ }^{3}$, education, income, self-reported health, smoking status, relationship status, health insurance, alcohol use, BMI category, exercise frequency, rurality, neighborhood socioeconomic score, aspirin use, atrial fibrillation, history of coronary artery disease, history of myocardial infarction, history of transient ischemic attack, diabetes status at baseline, systolic blood pressure, diastolic blood pressure, and $\mathrm{R}$ wave $\mathrm{aV} \mathrm{L}$ ).

'IPAW weight models included $L, C$, and $C *$; SACE attrition models included $L$ and $C$.

e Stabilized weights: race and $L$ (age, sex, and region) used in stabilization. 
disparity in stroke risk factors remained unchanged from estimates above.

\section{DISCUSSION}

Compared with white participants, black participants in REGARDS have increased risk of both incident hypertension and incident $\mathrm{LVH}$, with and without adjustment for all covariates in $L$ and $C$. These estimates of racial disparity are similar when employing complete-case, IPAW, and SACE analyses, indicating little evidence of selection bias in estimation of racial inequalities for these outcomes.

While our attrition model for death has reasonably high predictive ability, combination of all baseline predictors for the withdrawal model increased the $C$ statistic only marginally. Even inclusion of all pairwise interactions from backward selection increased these predictive measures only slightly. The $C$ statistics for death $(0.83)$ and withdrawal $(0.64)$ are very similar to those found in a recent study of smoking and cognitive decline, which reported $C$ statistics of 0.79 and 0.62 , respectively (15). This consistency is noteworthy because the study populations were quite different. We included more baseline behavioral and clinical measures in our models, but for the mortality model, the best individual predictors were age, income, and self-rated health. No measured variables were especially predictive of withdrawal from the study, although education had the highest unadjusted $C$ statistic. From an epidemiologic perspective, inability to predict study withdrawal using available social, behavioral, or clinical assessments gives some optimism that the withdrawal mechanism is random in nature rather than systematically related to outcomes.

One reason for the lack of difference between complete-case and IPAW analyses is likely due to none of the baseline predictors being notably predictive of study withdrawal. Although many of our predictors are subject to change after baseline measurement, these baseline variables have been shown to predict mortality and are expected to have enduring effects. However, there might exist an unmeasured confounder highly related to both race and these incident stroke risk factors that is causing selection bias. Nonetheless, REGARDS provides an unusually comprehensive list of likely predictors of study attrition. The relatively small range of nonstabilized weights observed, particularly for the study withdrawal model, is expected when measured baseline characteristics are not strongly predictive of attrition (37). Selection bias arises if attrition is influenced by both the exposure, or its determinants, and the outcome of interest or its determinants (4).

The usefulness of statistically creating artificial populations through IPAW, particularly those analyses that reflect no death over extended follow-up time, has drawn criticism as unrealistic to the natural life span, sometimes referred to as "up-weighting the dead" $(4,38)$. Both IPAW and SACE introduce a complexity in the analysis where multiple analytical decisions must be made, including which mechanisms for attrition should be considered, and which factors should be considered as predictors for each potential attrition mechanism. Loken and Gelman (39) argued that complex procedures introducing numerous decision points in which analysis is contingent on the data are undesirable because they lead to unrecognized multiple comparisons problems and render typical measures of uncertainty uninformative.
Use of estimated IPAWs to account for selection is subject to several assumptions, including positivity (nonzero probability of receiving every level of exposure for every combination of values of exposure and covariate histories that occur among individuals in the population) and correct model specification to estimate IPAWs. Assuming correct attrition-model specification, IPAW has been shown to be appropriate under the missing-at-random assumption. One study has presented simulation results indicating that IPAW performs adequately under the missing-not-atrandom assumption, provided the missingness model is correctly specified (40). Given that validity of the missingness models (equation 2) is a crucial assumption of IPAW, we investigated the impact of various modeling decisions on racial disparity estimates, finding little to no change in estimates (Web Table 2).

The regression model method for estimating SACE relies on several assumptions, enumerated above and in Tchetgen Tchetgen et al. (6) in extensive detail. The remarkable advantage to this approach is that SACE is unbiased under certain assumptions even when there are unmeasured common causes of selection bias and the outcome. Although the independence of unmeasured confounding $U$ and race conditional on $C$ is a relatively strong assumption, we have included many variables in $C$ through which $U$ might act. However, the existence of unmeasured confounders operating differently from those confounders considered here could introduce bias that we have not addressed here. It has been demonstrated mathematically and recently in published simulations that, under the right assumptions, selective attrition can introduce substantial bias in effect estimates. This bias occurs only when unmeasured confounders of selection and the outcome interact with race to affect persistence in the study $(4,13)$. The strength of association induced by such collider bias phenomena is often small and is constrained by the magnitude of the effect of exposure on attrition, the strength of the effect of the unmeasured common cause of attrition and survival, and whether the unmeasured factor and exposure interact on a multiplicative scale to determine continued study participation $(4,13,41,42)$. Although it might seem very likely that there are unmeasured confounders of selection and health outcomes not included in our analysis, the key question is the extent to which those factors interact with race to influence selection. Our analyses show that for a diverse set of social, behavioral, and clinical measures, the answer is: not to a great extent.

Attrition in REGARDS is not atypical compared with other studies of aging $(43,44)$, so it is extremely encouraging that we find limited evidence of selection bias. Nonetheless, many categories of measures, such as social network characteristics, literacy, and personality were not addressed here and seem plausible as unmeasured confounders of selection and health. We hope that future research prioritizes evaluating these domains and their potential to introduce selection bias. We found limited evidence of major multiplicative interactions with race and other factors in predicting either study withdrawal or mortality. It is therefore not surprising that the IPAW method did not alter results substantially. We cannot be sure whether the same observation would hold for any unmeasured common cause of attrition and our outcomes, but the similarity of SACE and complete-case analysis supports this implication.

From this large cohort study with moderate attrition over follow-up, we found no evidence of selection bias, despite 
substantial racial differences in attrition. Although no bias due to survival was observed, concerns about selection bias due to differential survival should nevertheless always be carefully considered and assessed. Few studies have comprehensive data to evaluate this issue empirically, and REGARDS is especially well-positioned to quantify any plausible impact of selection bias. Despite our extensive list of potential predictors of attrition, we have no evidence that accounting for survival altered our estimates of racial disparity in incidence of 2 important stroke risk factors.

\section{ACKNOWLEDGMENTS}

Author affiliations: Department of Biostatistics, School of Public Health, University of Alabama at Birmingham, Birmingham, Alabama (D. Leann Long, George Howard, Dustin M. Long, Suzanne Judd); Taub Institute for Research on Alzheimer's Disease and the Aging Brain, Columbia University Irving Medical Center, New York, New York (Jennifer J. Manly); Department of Neurology, Columbia University Irving Medical Center, New York, New York (Jennifer J. Manly); Department of Epidemiology and Biostatistics, Dornsife School of Public Health, Drexel University, Philadelphia, Pennsylvania (Leslie A. McClure); Department of Medicine, School of Medicine, University of Alabama at Birmingham, Birmingham, Alabama (Virginia G. Wadley); Division of General Internal Medicine, Cornell School of Medicine, New York, New York (Monika M. Safford); Kidney Research Institute, University of Washington, Seattle, Washington (Ronit Katz); and Department of Epidemiology and Biostatistics, University of California, San Francisco, San Francisco, California (M. Maria Glymour).

This research project is supported and cofunded by the National Institute of Neurological Disorders and Stroke and the National Institute on Aging (cooperative agreement U01 NS041588).

The content is solely the responsibility of the authors and does not necessarily represent the official views of the National Institute of Neurological Disorders and Stroke or the National Institute on Aging. Representatives of the National Institute of Neurological Disorders and Stroke were involved in the review of the manuscript but not directly involved in the collection, management, analysis, or interpretation of the data. We thank the other investigators, the staff, and the participants of the Reasons for Geographic and Racial Differences in Stroke study for their valuable contributions. A full list of participating Reasons for Geographic and Racial Differences in Stroke investigators and institutions can be found at http:// www.regardsstudy.org.

Conflict of interest: none declared.

\section{REFERENCES}

1. Seaman SR, White IR. Review of inverse probability weighting for dealing with missing data. Stat Methods Med Res. 2013;22(3):278-295.

2. Robins JM, Hernán MA, Brumback B. Marginal structural models and causal inference in epidemiology. Epidemiology. 2000;11(5):550-560.
3. Howe CJ, Cole SR, Lau B, et al. Selection bias due to loss to follow up in cohort studies. Epidemiology. 2016;27(1):91-97.

4. Hernán MA, Hernández-Díaz S, Robins JM. A structural approach to selection bias. Epidemiology. 2004;15(5): 615-625.

5. Kalaycioglu O, Copas A, King M, et al. A comparison of multiple-imputation methods for handling missing data in repeated measurements observational studies. J R Stat Soc Ser A Stat Soc. 2016;179(3):683-706.

6. Tchetgen Tchetgen EJ, Phiri K, Shapiro R. A simple regression-based approach to account for survival bias in birth outcomes research. Epidemiology. 2015;26(4): 473-480.

7. Glasser SP, Judd S, Basile J, et al. Prehypertension, racial prevalence and its association with risk factors: analysis of the REasons for Geographic And Racial Differences in Stroke (REGARDS) study. Am J Hypertens. 2011;24(2):194-199.

8. Howard G. Why do we have a stroke belt in the southeastern United States? A review of unlikely and uninvestigated potential causes. Am J Med Sci. 1999;317(3):160-167.

9. Howard VJ, Kleindorfer DO, Judd SE, et al. Disparities in stroke incidence contributing to disparities in stroke mortality. Ann Neurol. 2011;69(4):619-627.

10. Fujishiro K, Hajat A, Landsbergis PA, et al. Explaining racial/ ethnic differences in all-cause mortality in the Multi-Ethnic Study of Atherosclerosis (MESA): substantive complexity and hazardous working conditions as mediating factors. SSM Popul Health. 2017;3:497-505.

11. Kramer H, Han C, Post W, et al. Racial/ethnic differences in hypertension and hypertension treatment and control in the Multi-Ethnic Study of Atherosclerosis (MESA). Am J Hypertens. 2004;17(10):963-970.

12. Kochanek KD, Murphy SL, Xu J, et al. Mortality in the United States, 2013. NCHS Data Brief. 2014;(178):1-8.

13. Mayeda ER, Tchetgen Tchetgen EJ, Power MC, et al. A simulation platform for quantifying survival bias: an application to research on determinants of cognitive decline. Am J Epidemiol. 2016;184(5):378-387.

14. Cole SR, Hernán MA. Constructing inverse probability weights for marginal structural models. Am J Epidemiol. 2008; 168(6):656-664.

15. Weuve J, Tchetgen Tchetgen EJ, Glymour MM, et al. Accounting for bias due to selective attrition: the example of smoking and cognitive decline. Epidemiology. 2012;23(1):119-128.

16. Egleston BL, Scharfstein DO, Freeman EE, et al. Causal inference for non-mortality outcomes in the presence of death. Biostatistics. 2007;8(3):526-545.

17. Egleston BL, Scharfstein DO, MacKenzie E. On estimation of the survivor average causal effect in observational studies when important confounders are missing due to death. Biometrics. 2009;65(2):497-504.

18. Hayden D, Pauler DK, Schoenfeld D. An estimator for treatment comparisons among survivors in randomized trials. Biometrics. 2005;61(1):305-310.

19. Bhatt H, Safford M, Glasser S. Coronary heart disease risk factors and outcomes in the twenty-first century: findings from the REasons for Geographic And Racial Differences in Stroke (REGARDS) study. Curr Hypertens Rep. 2015;17(4):29.

20. East MA, Jollis JG, Nelson CL, et al. The influence of left ventricular hypertrophy on survival in patients with coronary artery disease: do race and gender matter? J Am Coll Cardiol. 2003;41(6):949-954.

21. Howard VJ, Cushman M, Pulley L, et al. The reasons for geographic and racial differences in stroke study: objectives and design. Neuroepidemiology. 2005;25(3):135-143. 
22. Howard VJ. Reasons underlying racial differences in stroke incidence and mortality. Stroke. 2013;44(6 suppl 1):S126-S128.

23. Kabagambe EK, Judd SE, Howard VJ, et al. Inflammation biomarkers and risk of all-cause mortality in the REasons for Geographic And Racial Differences in Stroke cohort. Am J Epidemiol. 2011;174(3):284-292.

24. Safford MM, Brown TM, Muntner PM, et al. Association of race and sex with risk of incident acute coronary heart disease events. JAMA. 2012;308(17):1768-1774.

25. Forman JP, Rimm EB, Stampfer MJ, et al. Folate intake and the risk of incident hypertension among US women. JAMA. 2005; 293(3):320-329.

26. Kaess BM, Rong J, Larson MG, et al. Aortic stiffness, blood pressure progression, and incident hypertension. JAMA. 2012; 308(9):875-881.

27. Gottesman RF, Albert MS, Alonso A, et al. Associations between midlife vascular risk factors and 25-year incident dementia in the Atherosclerosis Risk in Communities (ARIC) cohort. JAMA Neurol. 2017;74(10):1246-1254.

28. Sokolow M, Lyon TP. The ventricular complex in left ventricular hypertrophy as obtained by unipolar precordial and limb leads. Ann Noninvasive Electrocardiol. 2001;6(4):343-368.

29. Callahan CM, Unverzagt FW, Hui SL, et al. Six-item screener to identify cognitive impairment among potential subjects for clinical research. Med Care. 2002;40(9):771-781.

30. Hosmer DW, Lemeshow S, Sturdivant RX. Applied Logistic Regression. 3rd ed. Hoboken, NJ: Wiley; 2013.

31. Hinkley D. Transformation diagnostics for linear models. Biometrika. 1985;72(3):487-496.

32. Zou G. A modified Poisson regression approach to prospective studies with binary data. Am J Epidemiol. 2004;159(7):702-706.

33. Robins JM, Rotnitzky A. Semiparametric efficiency in multivariate regression models with missing data. J Am Stat Assoc. 1995;90(429):122-129.
34. Efron B, Tibshirani R. An Introduction to the Bootstrap. New York, New York: Chapman \& Hall; 1994.

35. Diez Roux AV, Merkin SS, Arnett D, et al. Neighborhood of residence and incidence of coronary heart disease. $N$ Engl J Med. 2001;345(2):99-106.

36. Howard VJ, McClure LA, Kleindorfer DO, et al. Neighborhood socioeconomic index and stroke incidence in a national cohort of blacks and whites. Neurology. 2016;87(22):2340-2347.

37. Vansteelandt S, Carpenter J, Kenward MG. Analysis of incomplete data using inverse probability weighting and doubly robust estimators. Methodology. 2010;6(1):37-48.

38. Chaix B, Evans D, Merlo J, et al. Commentary: weighing up the dead and missing: reflections on inverse-probability weighting and principal stratification to address truncation by death. Epidemiology. 2012;23(1):129-131.

39. Loken E, Gelman A. The statistical crisis in science. Am Sci. 2014;102(6):460.

40. Jones M, Mishra GD, Dobson A. Analytical results in longitudinal studies depended on target of inference and assumed mechanism of attrition. J Clin Epidemiol. 2015; 68(10):1165-1175.

41. Glymour MM, Vittinghoff E. Commentary: selection bias as an explanation for the obesity paradox: just because it's possible doesn't mean it's plausible. Epidemiology. 2014; 25(1):4-6.

42. Greenland S. Quantifying biases in causal models: classical confounding vs collider-stratification bias. Epidemiology. 2003;14(3):300-306.

43. Brilleman SL, Pachana NA, Dobson AJ. The impact of attrition on the representativeness of cohort studies of older people. BMC Med Res Methodol. 2010;10:71.

44. Weuve J, Proust-Lima C, Power MC, et al. Guidelines for reporting methodological challenges and evaluating potential bias in dementia research. Alzheimers Dement. 2015;11(9):1098-1109. 